\title{
MicroRNA as Upcoming Biomarkers for Psychiatric Conditions
}

\author{
Anoop Narahari*, Mariyah Hussain and Venkatesh Sreeram \\ University of Alabama, Department of Psychiatry and Behavioral sciences, Birmingham, Alabama, USA
}

\begin{abstract}
Neural derived exosomes can be used as a diagnostic marker to screen various psychiatric conditions. These intravenously injected exosomes carry the potential to cross the blood brain barrier and deliver miRNA specifically to neurons, microglia, and oligodendrocytes in the brain, resulting in a specific gene knockdown. MicroRNAs have been identified as markers in depression where miR-16 has been found to be a negative regulator of the serotonin transporter (SERT) through computer analysis. Futhermore, where miR 134 levels in bipolar disorder have been discovered to be inversely correlated with severity of manic symptoms; manipulating expression or activity of miR-219 can prove as a therapeutic tool for schizophrenia. Despite these studies, the exact nature and extent of dysregulation of microRNAs in psychiatric disorders is yet to be determined. To realize the therapeutic potential of MiRNAs in greater depth; efficient, tissue-specific and nonimmunogenic delivery of exosomes must be developed.
\end{abstract}

Keywords: MicroRNA(miR/miRNA); MessengerRNA (mRNA); Neuronal plasticity; Neurogenesis; Hippocampus; Depression; Bipolar disorder; Schizophrenia

\section{MicroRNAs as Upcoming Biomarkers for Psychiatric Conditions}

The intrinsic ability of synapses to adapt to environmental pressures, physiologic changes, and escaping the restrictions imposed by the genome is termed as neuronal/synaptic plasticity [1]. This includes molecular mechanisms and morphological changes such as altered neurotransmitter release, altered receptor expression, and alteration of dendritic size, shape, and density. These adaptive changes when dysregulated may be involved in the pathogenesis of psychiatric disorders.

In adult life, the effects of acute and chronic stress on neuronal plasticity are largely reversible,but early life stress induces persistent changes in neuronal plasticity that increase vulnerability to psychopathology such as depression and post-traumatic stress disorder (PTSD), suggesting the involvement of epigenetic mechanisms [2]. Particularly in neurons, epigenetic control seems important, since neurons are challenged with converting complex environmental stimuli into high-order functions. There are several mechanisms in which the environment interacts with the genome, including DNA methylation, histone modification, and microRNA regulation. A growing body of evidence demonstrates that microRNAs (miRNAs) are key players in such epigenetic regulation. Recently, circulating miRNAs are under intense investigation, as they bridge the current gap in our knowledge of the biology and effective treatment of these disabling disorders.

\section{What are MicroRNAs?}

MicroRNAs are important regulators of post-transcriptional gene expression. They may up- or down-regulate the translation of messenger RNA (mRNA) or render it unstable. A single mRNA may be regulated by multiple miRNAs, and, conversely, some miRNAs have the potential to target hundreds of mRNAs. MiRNAs primarily act to negatively regulate gene expression, miRNA over-expression should lead to down-regulation of their gene targets resulting in a negative correlation [3]. However, reciprocal relationships between miRNAs levels and their targets are emerging.

MiRNAs are approximately 22 nucleotide (nt)-long small noncoding RNAs. MiRNA biogenesis is initiated via transcription by RNA polymerase II, generating primary transcripts known as pri-miRs. PrimiRs are cropped by ribonuclease III Drosha and its cofactor, DiGeorge syndrome critical region gene 8 (DGCR8), to generate approximately 65 nt-long hairpin-shaped precursors known as pre-miRs [4].

Drosha and DGCR8 form a protein complex called a microprocessor, crucial for initial miR biogenesis. Pre-miRs are then exported by the nuclear transport factor exportin-5 (Exp5) to the cytoplasm where premiRNAs are processed by Dicer to a duplex of $\sim 22$ nucleotide doublestranded mature miRNAs [5]. One strand (antisense) of the mature miRNA is assembled into an RNA-induced silencing complex (RISC), while the other strand of the duplex is degraded [6]. One or both strands of a duplex have the potential to recognize cognate binding sites, miRNA recognition elements (MREs), in the 3'UTR of protein coding mRNA(s). Mature miRNAs silence gene expression by promoting translational repression and/or mRNA degradation. A single miRNA has the potential to target hundreds of distinct mRNA molecules.

Limbic area components including hippocampi, amygdale, and ventromedial prefrontal cortices are sensitive to stress-induced changes in neuronal plasticity; however, the hippocampi are the most sensitive amongst these. Elevation in glucocorticoids associated with activation of the HPA axis seems to be a central mechanism for regulation of neurogenesis by stress. The glucocorticoid receptor (GR) is a key regulator of structural plasticity and neurogenesis in the hippocampus. The highest density of glucocorticoid receptors (GRs) is present in these limbic structures, suggesting they are the primary targets of glucocorticoids in the brain in an acute stress response. Target genes of these miRNAs include those encoding for: brain-derived neurotrophic factor (BDNF), widely expressed throughout the brain where it promotes neuronal survival and maturation, synaptic plasticity and

*Corresponding author: Narahari A, University of Alabama, Department of Psychiatry and Behavioral sciences, Birmingham, Alabama, USA, E-mail: narahari@uab.edu

Received: August 31, 2015; Accepted: September 02, 2015; Published: Novomber 15, 2015

Citation: Narahari A, Hussain M, Sreeram V (2015) MicroRNA as Upcoming Biomarkers for Psychiatric Conditions. Clin Depress 1: 103.

Copyright: (c) 2015 Narahari A, et al.. This is an open-access article distributed under the terms of the Creative Commons Attribution License, which permits unrestricted use, distribution, and reproduction in any medium, provided the original author and source are credited. 
synaptic function; cAMP response element-binding protein (CREB), a transcription factor involved in synaptic plasticity and memory formation; and LIM domain kinase-1 (LimK-1), an enzyme that regulates dendrite size. MiRNA suppression of LimK-1 can be reversed by BDNF.

\section{MicroRNA as a marker in depression}

Due to the broad phenotype of Major depressive disorder, it is likely impossible to identify a single, effective diagnostic biomarker reflecting the activity of a pathway (or pathways) involved in depressive symptoms. MiR-16 was identified as a posttranscriptional repressor of the serotonin transporter (SERT), a pharmacological target of today's SSRIs and SNRIs. MiR-16 was identified as a negative regulator of the serotonin transporter (SERT) through computer analysis. The role of miRNAs in depression was initially ascertained from an in-vivo study of the raphe nuclei in mice; the investigators showed that fluoxetine increased levels of miR-16, which led to decreased SERT levels and increased serotonin signaling at the synapse. Hence, miR-16 acts as a central regulator of SERT expression. Furthermore, it also behaves as the mediator of the adaptive response of serotonergic and noradrenergic neurons to fluoxetine treatment [7]. MiRNAs provide a mechanism for adaptive changes in SERT expression in monoaminergic neurons, which can differentiate into either serotonergic (1C115-HT) or noradrenergic (1C11NE neuroectodermal cell line) neuronal cells.

\section{MicroRNA in bipolar disorder}

MiRNA-134 represses the translation of the Limk1-mRNA, a protein kinase that influences dendritic spine development. The miRNA-mediated repression of translation occurs by exogenous stimuli like BDNF, which has emerged as a key mediator for synaptic efficacy, neuronal connectivity and neuroplasticity [8]. In a study of 21 drugfree Bipolar Type 1 individuals with mania and 21 controls [9], miR134 levels in bipolar patients were inversely correlated with severity of manic symptoms, and its level increased following treatment. Although numbers were low, and treatment was open-label, this study suggests that miRNAs have the potential to serve as both therapeutic targets and biomarkers for bipolar disorder.

\section{MicroRNA in schizophrenia}

Chromosome 8p, from which at least seven miRNAs are transcribed, is an important area for neurodevelopmental disorders including autism and schizophrenia [10]. Patients with DiGeorge 22q11.2 deletion have a deficiency in DGCR8 (a key miRNA-processing gene) expression, resulting in decreased miR biosynthesis, imposing a 30-fold increased risk of schizophrenia [11]. The functional targets of these miRNAs include a number of genes that have been implicated in schizophrenia, such as BDNF, the dopamine receptor DRD1, the synaptic protein neuregulin 1 (NRG1) and early growth response gene 3 (EGR3).

Hypo-functional NMDA-receptor signaling in dorsolateral prefrontal cortex and superior temporal gyrus is consistent with cognitive and behavioral disturbances of schizophrenia, autism and attention deficit hyperactivity disorder (ADHD). MiR-219 negatively regulates the function of NMDA receptors, serving as an integral component of the NMDA-R signaling cascade. MiR-219 may directly modulate NMDA-R signaling by regulating the expression of components in this cascade. Over-expression of miR-219 in cortical cells may inhibit $\mathrm{Ca}^{2+}$ influx through NMDA-R signaling transduction upon receptor activation in vitro. MiR-219 targets the calcium/calmodulindependent protein kinase II $\gamma$ subunit (CaMKII $\gamma$ ), a component of the NMDA receptor signaling cascade [12]. This microRNA responds rapidly to alterations in NMDA-R signaling, exerts translational control of CaMKII expression, and contributes to altered behavioral manifestations. Manipulating the expression or activity of miR-219 may therefore provide a potential therapeutic tool for the treatment of schizophrenia.

The most well-studied miRNA family is that of miR-132/miR-212. These miRNAs share sequence similarities and are expressed from the same genomic location. Another notable similarity between miR-219 and miR-132 is that they were both shown to influence genes important for circadian clock entrainment [13], which is of potential relevance to schizophrenia given the observed deficits in circadian synchronization $[14,15]$.

\section{Conclusion}

Neural miRNAs are responsive to environmental, synaptic, and pathological changes and are actively secreted by cells as exosomes from brain into blood. While serum miRs cannot cross the blood-brain barrier (BBB), naturally occurring exosomes are capable of crossing the BBB. These exosomes bear cell-type specific surface markers. Using a neural-specific surface marker, neural-derived exosomes can be successfully isolated and can be used as a diagnostic marker to screen many psychiatric conditions. For example, brain-derived exosomes are fused with neuron-specific RVG peptide (Rabies virus glycoprotein, a peptide known to selectively target the nicotinic acetylcholine receptor in neurons), and are loaded with MiRNA by electroporation. These intravenously-injected exosomes, crossing the BBB, deliver MiRNA specifically to neurons, microglia and oligodendrocytes in the brain, resulting in a specific gene knockdown. However, to realize the therapeutic potential of MiRNAs in greater depth, efficient, tissuespecific and nonimmunogenic delivery of exosomes must be developed. RVG exosomes are capable of delivering MiRNAs specifically and safely after systemic administration and therefore represent a promising vehicle for gene therapies targeting psychiatric disorders. There continues to be a search to uncover mechanisms of the development of neuropsychiatric diseases and to generate more successful therapeutic strategies. The exact nature and extent of dysregulation of microRNAs in psychiatric disorders is yet to be determined. Current studies are heterogeneous, and even studies of same source material have delivered disparate findings. Considerable obstacles remain before microRNAs can be used as therapeutic targets in psychiatric disorders.

\section{References}

1. Pascual-Leone A, Amedi A, Fregni F, Merabet LB (2005) The plastic human brain cortex. Annual review of neuroscience 28: 377-401

2. Schouten M, Aschrafi A, Bielefeld P, Doxakis E, Fitzsimons C (2013) MicroRNAs and the regulation of neuronal plasticity under stress conditions. Neuroscience 241: 188-205.

3. Wang X, Wang X (2006). Systematic identification of microRNA functions by combining target prediction and expression profiling. Nucleic acids research 34: $1646-52$

4. Lee Y, Ahn C, Han J, Choi H, Kim J, et al. (2103) The nuclear RNase III Drosha initiates microRNA processing. nature 425(6956): 415-9.

5. Yi R, Qin Y, Macara IG, Cullen BR (2103) Exportin-5 mediates the nuclear export of pre-microRNAs and short hairpin RNAs. Genes \& development 17: 3011-6.

6. Schwarz DS, Hutvágner G, Du T, Xu Z, Aronin N, et al (2103) Asymmetry in the assembly of the RNAi enzyme complex. Cell 115: 199-208.

7. Baudry A, Mouillet-Richard S, Schneider B, Launay J-M, Kellermann O (2010) miR-16 targets the serotonin transporter: a new facet for adaptive responses to antidepressants. Science 329(5998): 1537-41. 
8. Schratt GM, Tuebing F, Nigh EA, Kane CG, Sabatini ME, et al. (2006) A brainspecific microRNA regulates dendritic spine development. Nature 439(7074): 283-9.

9. Rong H, Liu TB, Yang KJ, Yang HC, Wu DH, et al. (2011) MicroRNA-134 plasma levels before and after treatment for bipolar mania. Journal of psychiatric research 45: 92-5.

10. Tabares-Seisdedos R, Rubenstein J (2009) Chromosome 8p as a potential hub for developmental neuropsychiatric disorders: implications for schizophrenia, autism and cancer. Molecular psychiatry 14: 563-589.

11. Fénelon K, Mukai J, Xu B, Hsu P-K, Drew LJ, et al (2011) Deficiency of Dgcr8, a gene disrupted by the 22q11. 2 microdeletion, results in altered short-term plasticity in the prefrontal cortex. Proceedings of the National Academy of
Sciences 108: 4447-52.

12. Kocerha J, Faghihi MA, Lopez-Toledano MA, Huang J, Ramsey AJ, et al (2009) MicroRNA-219 modulates NMDA receptor-mediated neurobehaviora dysfunction. Proceedings of the National Academy of Sciences 106: 3507-12.

13. Cheng H-YM, Papp JW, Varlamova O, Dziema H, Russell B, et al. (2007) microRNA modulation of circadian-clock period and entrainment. Neuron 54 : 813-29.

14. Boivin DB (2010) Circadian rhythms and clock genes in psychotic disorders. The Israel journal of psychiatry and related sciences $47: 27-35$.

15. Lamont EW, Legault-Coutu D, Cermakian N, Boivin DB (2007) The role of circadian clock genes in mental disorders. Dialogues in clinical neuroscience 9: $333-42$ 